Bull. Mater. Sci., Vol. 3, Number 4, December 1981, pp. 415-422. (C) Printed in India.

\title{
Galvanomagnetic properties of plastically deformed InSb single crystals
}

\author{
M NAGABHOOSHANAM and V HARI BABU \\ Solid State and Materials Science Laboratories, Department of Physics, \\ Osmania University, Hyderabad 500 007, India
}

MS received 29 December 1980; revised 26 March 1981

\begin{abstract}
The dc Hall effect, dc conductivity and mobility have been studied on deformed and undeformed samples of $n$-type InSb from liquid nitrogen temperature to room temperature. These studies have shown that the Hall coefficient values of deformed samples do not differ much from undeformed sample, but a considerable amount of change was observed in mobility, suggesting that equal number of donor and acceptor type dislocations are introduced during the deformation process. In addition the mobility variation of the deformed samples with temperature has shown a peak in $170-300^{\circ} \mathrm{K}$ range. The dislocation mobility $\left(\mu_{D}\right)$ is deduced from the observed mobilities of deformed and undeformed samples. The plot $\mu_{D} v s T$ has two regions, region 1 being independent of temperature and region 2 having a linear increase with temperature. The $\beta$ factor obtained from region 2 is found to be almost equal to the one calculated from Dexter and Seitz model. The dislocation densities at room temperature are also calculated for the deformed samples using the above model.
\end{abstract}

Keywords. dc Hall coefficient; dc conductivity; mobility; deformation; dislocations; dislocation mobility.

\section{Introduction}

It is a well-known fact that plastic deformation alters the electrical properties of semiconductors. For example, deformation in silicon (Zhurkin et al 1967; Staunton and Pollak 1967) and Germanium (Komatsubara and Tagsugi 1965; Cuevas and Fritzsche 1967; Fritsche 1962) has produced changes in the carrier mobility, life time and carrier concentration by orders of magnitude. Recently (Yasuda et al 1978) have established that uniaxial stress will also produce change in the dielectric properties of anti-ferroelectric materials. InSb, which can be easily grown into single crystals, was subjected to plastic deformation and influences of magnetic field and temperature on galvanomagnetic properties were studied (Kaila 1977; Galvanov and Obuknov 1973). This semiconductor on slight deformation produces an appreciable density i.e., about $10^{7}$ dislocations $/ \mathrm{cm}^{2}$ (Kaila 1977). Furthermore the behaviour of certain galvanomagnetic coefficients are very sensitive to the presence of the dislocations. Recently the authors (Nagabhooshanam and Hari Babu 1980) have studied the effect of dislocations on galvanomagnetic properties of InSb single crystals, dislocations being created during the annealing process. In the present work we report dc Hall coefficient and dc conductivity measurements done from liquid nitrogen temperature to room temperature on $n$-type InSb crystals before and after applying anisotropic 
uniaxial stress along [111] direction. The stress was applied with a diamond indentor at regular intervals of distance on both sides of the $n$-InSb single crystal at room temperature. The mobility due to the scattering of charge carriers by the dislocations has been found to be temperature independent till a particular temperature, and increases linearly thereafter. The temperature coefficient of the dislocation scattering mobility for the second region was determined and compared with the value determined by using Dexter and Seitz model (Dexter and Seitz 1952) for stationary edge dislocations. These two values are almost comparable.

\section{Experimental details}

The $n$-InSb crystals were obtained from Electronics Corporation of India Limited, Hyderabad. Slices perpendicular to the $c$-axis were cut from the boule. From these slices, samples of rectangular bar geometry of about $8 \times 4 \times 0.75 \mathrm{~mm}^{3}$ were cut and then lapped with fine grains of carborandom. The work damage at the surface during sawing and lapping was removed by chemical etching. The etchant consisted of 40 parts lactic acid +10 parts $\mathrm{HNO}_{3}+5$ parts $\mathrm{HF}+15$ parts $\mathrm{H}_{2} \mathrm{O}$ and the time of etching was about 30 secs. To the dried crystal five ohmic contacts were made ( 2 current contacts and 3 voltage contacts) with dots of high purity indium at $200^{\circ} \mathrm{C}$ in a $10^{-5}$ torr vacuum. The size of indium contacts was about $0.5 \mathrm{~mm}$ in diameter while the current contacts were spread over the entire cross-section of the sample.

After observing the Hall coefficient and conductivity with temperature the sample was indented at the room temperature with a diamond pointer attached with the Universal Microscope (Carl Zeiss, Zena) to produce plastic deformation. The load applied was $100 \mathrm{~g}$ and the time of indentation being $10 \mathrm{sec}$. each. For every 50, 100 and 150 indentations the observations were made from liquid nitrogen temperature $\left(77^{\circ} \mathrm{K}\right)$ to the room temperature $\left(300^{\circ} \mathrm{K}\right)$. Care was taken to see that indentations made are equidistant. This method of producing plastic deformation avoids removal of ohmic contacts during deformation process.

Five probe technique was used to measure the Hall coefficient $\left(R_{H}\right)$ and conductivity $(\sigma)$ of the samples, one probe being kept common for the measurements of $R_{H}$ and $\sigma$. Measurements were made from liquid nitrogen temperature to the room temperature by mounting the sample on a copper block (with electrical insulation) in a cryostat in which heating of the sample was achieved by conduction through the copper block. The cryostat is kept exactly in the middle of the pole caps of the 6 inch electromagnet and it was made sure that the sample is under the action of a uniform magnetic field. The magnetic field was measured by a differential Gaussmeter. The Hall voltage and the voltage across the current leads were measured by means of a Phillips dc microvoltmeter at different currents through the sample. Every time the direction of the current through the sample and the direction of the magnetic field were changed, and the Hall voltages were measured. The average of these four measurements is taken for the Hall coefficient calculations. The temperature measurements were made by means of a copper constantan thermocouple, one of its junction being kept at $0^{\circ} \mathrm{C}$. 


\section{Results and discussion}

The variation of Hall coefficient with temperature $\left(\log R_{H} v s 1 / T\right)$ for the four $n$-type InSb samples, having indentations zero (pure), 50, 100 and 150 (hereafter referred as sample S1, S2, S3 and S4) are shown in figure 1 corresponding to a magnetic field of $7.5 \mathrm{kG}$. It is seen from the figure that the $R_{H}$ values of $\mathrm{S} 2, \mathrm{~S} 3$ and $\mathrm{S} 4$ do not differ much from $S 1$. It is also seen that $R_{H}$ value decreases slowly with the increase in temperature up to a certain temperature and thereafter decreases rapidly. This behaviour is typical for a degenerate extrinsic semiconductor.

Figure 2 shows the temperature dependence of conductivity of S1, S2, S3 and S4. It is seen that there is a considerable amount of change in the conductivity due to deformation.

The variation of mobility with temperature for the four samples is shown in figure 3. It is observed that the electron mobility decreases steadily as the deformation progresses. The mobility difference between deformed and undeformed crystals is more at liquid nitrogen temperature and is less at room temperature.

The near constancy in the values of $R_{H}$, in the extrinsic region and the difference in mobility between deformed and undeformed crystals indicate that the donor and acceptor concentrations introduced during deformation process are almost equal as

(i) the Hall coefficient varies inversely with the charge carrier concentration ( $n$ ) and $n=N_{D}-N_{A}$, where $N_{D}$ and $N_{A}$ are donor and acceptor concentrations.

(ii) mobility is more closely related to the total impurity concentration $\left(N_{I}\right)$ i.e. $N_{I}=N_{D}+N_{A}$.

These dislocations are nothing but the rows of atoms with unsaturated dangling bonds on the edges of extra half planes created throughout the body of the crystal. When

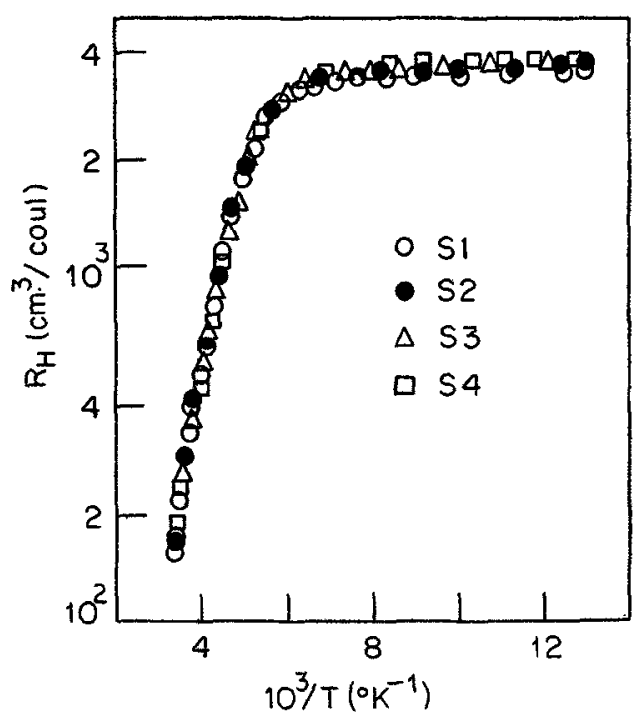

Figure 1. Temperature variation of dc Hall coefficient $\left(\log R_{H} v_{S} 1 / T\right)$ for the four samples S1, S2, S3 and S4 of $n$-type InSb. 


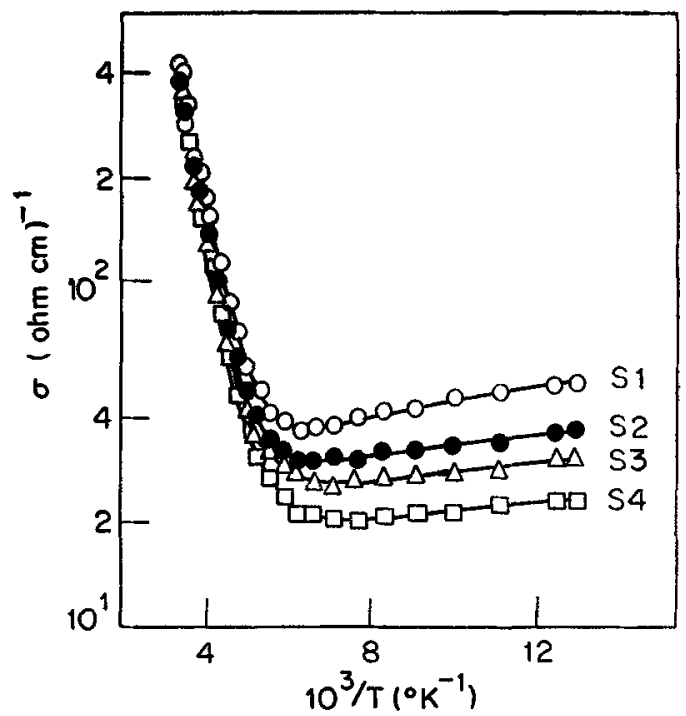

Figure 2. Temperature variation of the dc conductivity $(\log \sigma$ is $1 / T)$ for the four samples S1, S2, S3 and S4 of $n$-type InSb.

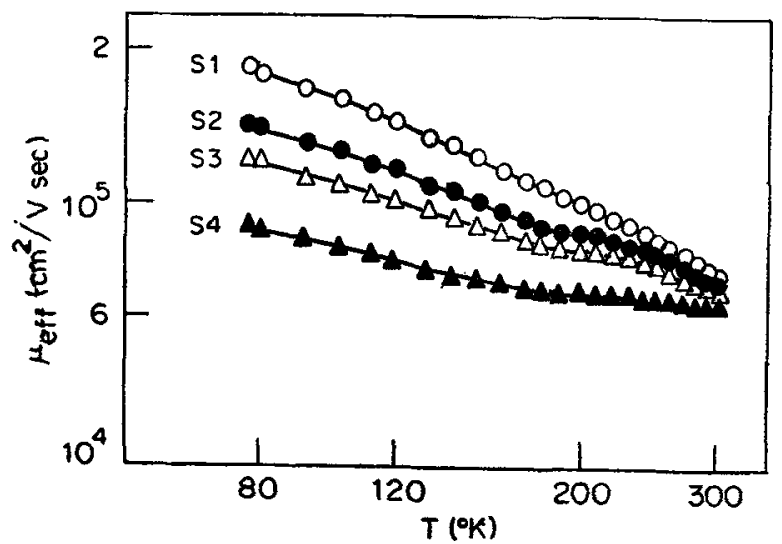

Figure 3. Temperature dependence of the observed Hall electron mobility $\left(\log \mu_{\text {eff }}\right.$ vs $\log T$ for $\mathrm{S} 1, \log \mu_{\text {eff }}^{\prime}$ vs $\log T$ for $\mathrm{S} 2, \mathrm{~S} 3$ and $\mathrm{S} 4$ ) in the four samples $\mathrm{S} 1, \mathrm{~S} 2, \mathrm{~S} 3$ and $S 4$. In sample $S 1$, maxima is not observed.

In atoms are at the edge of the extra half planes, there are no excess of electrons and such a row will create an acceptor level in the forbidden gap. When $\mathrm{Sb}$ atoms form these rows, each atom has a saturated electron pair and the dislocations should introduce a donor level. These dislocations are called respectively In and Sb type and have been effectively observed to exhibit both donor and acceptor behaviour (Bell et al 1960; Duga 1962). It is also possible that a small amount of trapping at these dislocations may occur and is observable only in the specimens of higher purity. This effect was found to be of only $1 \%$ in specimens containing $1 \times 10^{14}$ charge carriers per $\mathrm{cm}^{3}$ (Duga et al 1959). As our original sample ( $\mathrm{S} 1$ ) contains carrier concentration of $1.6 \times 10^{18} \mathrm{~cm}^{3}$, this effect can almost be neglected. 
It is also seen from figure 3 that the mobility corresponding to samples $S 2, \mathrm{~S} 3$ and S4 show slight maximum in the range $170 \sim 300^{\circ} \mathrm{K}$ which was also observed by Duga in uniaxially compressed bulk InSb (Duga et al 1959) and by Wieder in InSb thin films prepared by flash evaporation (Wieder 1966). This maximum was not observed in the undeformed sample $\mathbf{S 1}$.

The temperature dependence of the electron mobility in these samples will be interpreted in terms of the model proposed by Dexter and Seitz according to which, scattering of charge carriers by the deformation potential associated with stationary edge dislocations is possible. For simplicity the polar nature of the InSb lattice, electron hole scattering as well as the impurity scattering will be neglected. Lattice scattering will be characterized by a mobility $\mu_{L}$ and a relaxation time $\tau_{L}$ and dislocation scattering will be represented by a mobility $\mu_{D}$ and a relaxation time $\tau_{D}$. The experimentally measured mobility $\mu_{\text {eff }}$ is related to $\mu_{L}$ and $\mu_{D}$ by

$$
\frac{1}{\mu_{\text {eff }}}=\frac{1}{\mu_{L}}+\frac{1}{\mu_{D}}
$$

The value of $\mu_{D}$ is given by

$$
\mu_{D}=\left(e / m^{*}\right) \tau_{D}
$$

where $m^{*}$ is the effective mass of the charge carriers and $\tau_{D}$ is defined as (Wieder 1966)

$$
\tau_{D}=\frac{32}{3 \pi}\left(\frac{1-\nu}{1-2 \nu}\right)^{2} \frac{k T h}{\epsilon^{2} \lambda^{2} N}
$$

The parameters involved and their values in (3) are given in table 1. Substituting the equation for lattice mobility

$$
\mu_{L}=1.09 \times 10^{9} \times T^{-1.68}
$$

\begin{tabular}{|c|c|c|c|}
\hline & Parameters & Values taken & Reference \\
\hline $\begin{array}{l}\epsilon \\
v \\
\lambda\end{array}$ & $\begin{array}{l}=\text { Energy associated with the dislocation } \\
=\text { Poisson's ratio } \\
=\text { Lattice spacing }\end{array}$ & $\begin{array}{l}6.4 \mathrm{eV} \\
0.4 \\
6.48 \AA\end{array}$ & $\begin{array}{l}\text { Ehrenreich } 1957 \\
\text { Wieder } 1966 \\
\text { Wieder } 1966\end{array}$ \\
\hline$m_{n}^{*}$ & $=$ Electron effective mass & $0.0139 \mathrm{~m}_{0}$ & Johnson and Dickey 1970 \\
\hline$m_{p}^{*}$ & $=$ Hole effective mass & $0.42 \mathrm{~m}_{8}$ & Pidgeon and Brown 1966 \\
\hline $\begin{array}{l}T \\
N \\
h \\
k\end{array}$ & $\begin{array}{l}=\text { Absolute temperature } \\
=\text { Dislocation density } \\
=\text { Planck's constant } \\
=\text { Boltzmann constant }\end{array}$ & $\begin{array}{l}1.0546 \times 10^{-27} \mathrm{erg} \mathrm{sec} \\
8.615 \times 10^{-5} \mathrm{eV} /{ }^{\circ} \mathrm{K}\end{array}$ & \\
\hline
\end{tabular}

Table 1. Parameters involved in equation (3) and their values. 
as given by Hrostowski (Hrostowski et al 1955) and (2) and (3) in (1), one gets

$$
\frac{1}{\mu_{\mathrm{eff}}}=\frac{T^{1.68}}{1.09 \times 10^{9}}+\frac{1}{\beta T}
$$

where

$$
\beta=\frac{32}{3 \pi}\left(\frac{e}{m^{*}}\right)\left(\frac{1-\nu}{1-2 v}\right)^{2} \frac{k h}{\epsilon^{2} \lambda^{2} N}
$$

Taking the derivative of (5) with respect to $T$, maximizing the resultant expression and solving for $\beta$, we get

$$
\beta=6.49 \times 10^{8} T_{0}^{2.88},
$$

where $T_{0}$ is the peak temperature in the mobility versus temperature plot. The peak temperature values and the calculated $\beta$ values of the deformed samples are tabulated in table 2 .

For each of the three specimens $\mathbf{S} 2, \mathrm{~S} 3$ and $\mathrm{S} 4, \mu_{D}$ as a function of temperature is calculated using the measured mobility values of deformed $\left(\mu_{\text {eff }}^{\prime}\right)$ and undeformed $\left(\mu_{\text {eff }}\right)$ samples using the relation

$$
\frac{1}{\mu_{\mathrm{eff}}^{\prime}}=\frac{1}{\mu_{\mathrm{eff}}}+\frac{1}{\mu_{D}} \text {. }
$$

The variation of $\mu_{D}$ with temperature can be seen in figure 4. For samples $\mathbf{S 2}, \mathbf{S 3}$ and S4, $\mu_{D}$ variation has two regions, region 1 which extends from $77^{\circ} \mathrm{K} \sim 170^{\circ} \mathrm{K}$, where $\mu_{D}$ is almost constant and region 2 from $170^{\circ} \mathrm{K}$ to room temperature where $\mu_{D}$ increases linearly. Experimentally measured values of $\beta$ from the region 2 are also given in table 2. The arrived values of $\beta$ using (7) more or less agree with the experimental values. The slight difference in them is because of the initial assumptions.

From (2) and (3) we get the dislocation density as

$$
N=\frac{32}{3 \pi}\left(\frac{1-\nu}{1-2 v}\right)^{2} \frac{k T h e}{\epsilon^{2} \lambda^{2} \mu_{D} m^{*}},
$$

Table 2. Peak temperatures, values of $\beta$ obtained by two methods, dislocation mobility and dislocation density of three samples $S 2, S 3$ and $S 4$.

\begin{tabular}{cccccc}
\hline $\begin{array}{c}\text { Sample } \\
\text { No. }\end{array}$ & $\begin{array}{c}\text { Measured } \\
T_{0}\end{array}$ & $\begin{array}{c}\text { From } \\
\text { Equation (7) }\end{array}$ & $\begin{array}{c}\text { From } \\
\text { region } 2 \text { of } \\
\text { figure } 4\end{array}$ & $\begin{array}{c}\mu D\left(\mathrm{~cm}^{2} / \mathrm{V}-\mathrm{sec} /{ }^{\circ} \mathrm{K}\right) \\
\text { at } 300^{\circ} \mathrm{K}\end{array}$ & $\begin{array}{c}N(\mathrm{sec}) \\
\text { at } 300^{\circ} \mathrm{K}\end{array}$ \\
\hline S2 & 190 & $5.07 \times 10^{2}$ & $6.12 \times 10^{2}$ & $1.2934 \times 10^{6}$ & $2.9668 \times 10^{8}$ \\
S3 & 225 & $3.22 \times 10^{2}$ & $3.04 \times 10^{2}$ & $7.4684 \times 10^{5}$ & $5.1380 \times 10^{8}$ \\
S4 & 280 & $1.79 \times 10^{2}$ & $1.36 \times 10^{2}$ & $4.3452 \times 10^{5}$ & $8.8311 \times 10^{8}$ \\
\hline
\end{tabular}




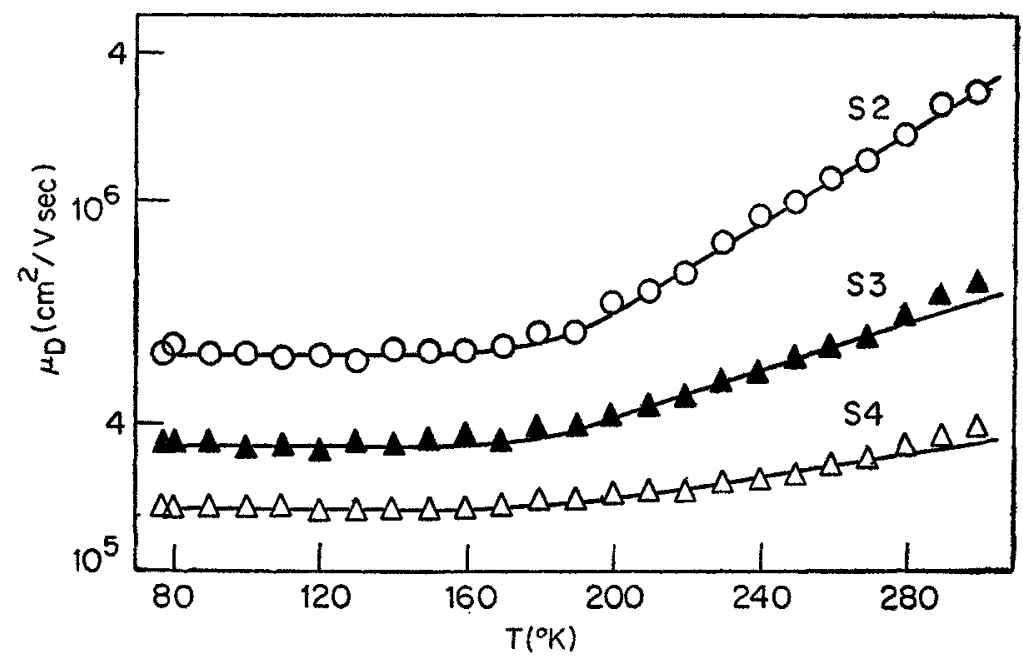

Figure 4. Temperature dependence of the dislocation mobility $\left(\mu_{D} v S T\right)$ of the three samples S2, S3 and S4. Two regions 1 and 2 are seen. Region 1 is independent of temperature and region 2 has a linear variation with temperature.

and substituting the value of $\mu_{D}$ at room temperature in this equation, dislocation densities have been calculated for the three samples and are given in table 2. Their order of magnitude are same as those observed by Duga (1962). Finally it is concluded that by this simple method of plastic deformation one can introduce dislocations in a controlled manner and study the effect of dislocations on galvanomagnetic properties.

\section{Acknowledgements}

One of the authors (MNB) acknowledges the UGC; New Delhi for the award of a Fellowship. The authors also thank the Head, Department of Physics for his interest in this work and encouragement.

\section{References}

Bell R L, Latkowski R and Willoughby A F W 1960 J. Mater. Sci. 166

Cuevas M and Fritzsche H 1967 Phys. Rev. 1371847

Dexter D L and Seitz F 1952 Phys. Rev. 86964

Duga J J, Willardson R K and Beer A C 1959 J. Appl. Phys. 301798

Duga J J 1962 J. Appl. Phys. 33169

Ehrenreich E J 1957 Phys. Chem. Solids 2131

Fritsche H 1962 Phys. Rev. 1251552

Galvanov V V and Obuknov S A 1973 Sov. Phys. Semicond. (Engl. Transl.) 7287

Hrostowski H J, Morin F J, Geballe T H and Wheatley G H 1955 Phys. Rev. 1001672

Johnson E J and Dickey D H 1970 Phys. Rev. B1 2676

Kaila M M 1977 Phys. Status Solidi A44 K135

Komatsubara K and Tagsugi N 1965 J. Phys. Soc. Jpn. 201790

Nagabhooshanam M and Hari Babu V 1980 J. Appl. Phys. 513250 
Pidgeon C R and Brown R.N 1966 Phys. Rev. 146575

Staunton H F and Pollak F H 1967 Phys. Lett. A25 751

Wider H H 1966 Solid-State Electron. 9373

Yasuda N, Shimizu H and Fujimoto S 1978 J. Appl. Phys. 49383

Zhurkin B G, Kucherenko I V and Penin N A 1967 Sov. Phys. Solid State (Engl. Transl.) 82767 